\title{
Comparison of ultrasound-assisted and pure fluoroscopy-guided extracorporeal shockwave lithotripsy for renal stones
}

Tsung-Hsin Chang ${ }^{1 *}$, Wun-Rong Lin ${ }^{1,2}$, Wei-Kung Tsai ${ }^{1,2}$, Pai-Kai Chiang ${ }^{1,2}$, Marcelo Chen ${ }^{1,2,3}$, Jen-Shu Tseng ${ }^{1 *}$ and Allen W. Chiu ${ }^{1,4}$

\begin{abstract}
Background: In this study, we aimed to compare the efficacy and clinical outcomes of shock wave lithotripsy (SWL) for patients with renal stones using pure fluoroscopy (FS) or ultrasound-assisted (USa) localization with two lithotripters.

Methods: We retrospectively identified 425 patients with renal calculi who underwent SWL with either a LiteMed LM-9200 ELMA lithotripter (209 cases), which combined ultrasound and fluoroscopic stone targeting or a Medispec EM-1000 lithotripter machine (216 cases), which used fluoroscopy for stone localization and tracking. The patient demographic data, stone-free rates, stone disintegration rates, retreatment rates and complication rates were analyzed.
\end{abstract}

Results: The USa group had a significantly higher overall stone-free rate (43.6 vs. $28.2 \%, p<0.001)$ and stone disintegration rate ( 85.6 vs. $64.3 \%, p<0.001)$, as well as a significantly lower retreatment rate $(14.8$ vs. $35.6 \%, p<0.001)$ and complication rate ( $1.9 \mathrm{vs} .5 .5 \%, \mathrm{p}=0.031$ ) compared with the $\mathrm{FS}$ group. This superiority remained significant in the stone size $<1 \mathrm{~cm}$ stratified group. In the stone size $>1 \mathrm{~cm}$ group, the stone-free rate (32.4 vs. $17.8 \%, p=0.028)$, disintegration rate ( 89.2 vs. $54.8 \%, p=0.031)$ and retreatment rate ( 21.6 vs. $53.4 \%, p<0.001)$ were still significantly better in the USa group, however there was no significant difference in the complication rate. The most common complication was post-SWL-related flank pain.

Conclusion: SWL is a safe and non-invasive way of treating renal stones. This study compared two electromagnetic shock wave machines with different stone tracking systems. LiteMed LM-9200 ELMA lithotripter, which combined ultrasound and fluoroscopic stone targeting outperformed Medispec EM-1000 lithotripter, which used fluoroscopy for stone localization and tracking, with better stone-free rates and disintegration rates, as well as lower retreatment rates and complications with possible reduced radiation exposure.

Keywords: Shock wave lithotripsy, Nephrolithiasis, Kidney, Ultrasonography

*Correspondence: KeithChang0910@gmail.com; urojst@gmail.com 1 Department of Urology, MacKay Memorial Hospital, No. 92, Sec. 2, Zhongshan N. Rd., Taipei City 10449, Taiwan

Full list of author information is available at the end of the article

\section{Background}

Extracorporeal shock wave lithotripsy (SWL) has been used as a non-invasive method of urolithiasis management since the 1980's [1]. It has been proven to be a safe, efficient way of treating non-infected upper urinary tract stones and remains one of the most commonly used treatment modality for renal stones worldwide today. 
Contemporary studies have shown that the success rate of SWL for renal stones ranges from 47 to $92 \%$ [2-7]. This highly variable result is due to various crucial factors, including stone composition and size [8-11], skin to stone distance [12], lithotripter energy power [13] and frequency [14, 15], patient positioning [16], and patient tolerance and respiration $[16,17]$. Less than half of the administered shockwaves may be accurately focused on the targeted calculus [18], and the excessive energy and shockwaves can cause damage to the parenchyma and adjacent organs. Chen et al. [19] demonstrated increased accuracy of stone targeting by using an electromagnetic lithotripter integrated with both ultrasound real-time tracking system and traditional fluoroscopy (LiteMed LM-9200 ELMA). Through the alternative use of ultrasound and fluoroscopy, the energy of the lithotripter may be more focused on the target stone during the whole session, thus increasing SWL efficiency. However, only a few studies have compared the efficacy of ultrasoundbased lithotripters with fluoroscopy-based lithotripters. It is difficult to compare efficacy between different institutions due to variabilities in treatment protocols and operators. The current study compared the efficacy and clinical outcomes of two electromagnetic lithotripters using different stone localization methods (LiteMed LM-9200 ELMA lithotripter: combined ultrasound and fluoroscopic stone targeting; Medispec EM-1000 lithotripter: pure fluoroscopic stone targeting), in a single center with 2 hospital branches, using the same group of urologists and the same set of patients and surgical indications. The machines were operated by the same two experienced technicians, which minimizes operator and institutional related bias.

The aim of the current study was to compare the efficacy and clinical outcomes of these two different stone localization modalities in the treatment of renal stones in one single medical center.

\section{Methods}

The present study retrospectively reviewed the medical records of patients with radio-opaque kidney stones with stone size between 0.5 to $2.0 \mathrm{~cm}$ who underwent SWL in a single medical center with 2 hospital branches between January 2013 and March 2019. Exclusion criteria were patients with congenital anomalies or urinary diversion, pediatric patients or patients who received SWL as a combination therapy with other treatment modalities. Patients with radiolucent stones were also excluded as plain $\mathrm{x}$-rays were used as the standard follow-up modality.

All patients underwent imaging and laboratory studies prior to SWL. Plain abdominal radiography of the kidneys, ureter and bladder (KUB), renal sonography, intravenous urography (IVU) and abdominal computed tomography $(\mathrm{CT})$ were used to diagnose and identify the stones. The size of the stones were measured on the KUB using maximal stone diameters.

Patients underwent SWL at two branches of our medical center with either a LiteMed LM-9200 ELMA lithotripter or Medispec LTD EM-1000 lithotripter, depending on their location. The distance between the two branches of our medical center were about $10 \mathrm{~km}$ with about a 20 min driving time and both were located in the northern part of the city. There were no regional characteristics different between the patients of the two branches, such as water composition and eating habits. The energy source of both lithotripters was an electromagnetic shockwave source. The focal length was $145 \mathrm{~mm}$ for EM-1000 and $125 \mathrm{~mm}$ for LM-9200. All SWL treatments were performed on an ambulatory basis by the same group of urologists. Both machines were operated by the same two experienced technicians. The LiteMed LM-9200 ELMA lithotripter used ultrasonography and fluoroscopy for dual focusing of stone localization and tracking, while the EM1000 machine used only fluoroscopy for stone visualization, with an integrated computerized fluoroscopic X-ray (Visionspec ${ }^{\mathrm{TM}}$ ). The LiteMed LM-9200 machine has a auto-firing system, the shockwaves will only be fired when the stone is in the focal zone.

The same standardized SWL protocol was used for both machines except for the stone localization method. The patient was placed in a supine position without a restraint belt. The cushion size and lubricating jelly used were the same in both branch. and the stone was located by the fluoroscopy in both group. In the USa group, ultrasound was combined use for dual stone localization before initiating the SWL session and during the treatment for real time tracking of the stone. While in the FS group, only pure fluoroscopy was used throughout the whole session. During SWL, the initial energy level was set at $14 \mathrm{KV}$ and could gradually increased up to $19 \mathrm{kV}$ if the stones did not show adequate fragmentation under low energy settings. The shock wave rate was set at 60 shocks per minute for both lithotripters. The maximum number of shockwaves delivered in one session was 3,000. Light intravenous propofol and dormicum were used as analgesics or for sedation if the patient suffered from pain or could not tolerate lying still in the supine position throughout the SWL session. The patient's position was adjusted throughout the SWL session for better stone visualization. If the patient's stone is still in focal zone, the position of the patient will not be changed to avoid constant de-coupling and re-coupling. In the FS group, the position was checked and adjusted after every 600 shocks or when the patient moved. While in the USa 
group, positioning was adjusted based on the ultrasound tracking of the stone location throughout the SWL session. All patient received SWL sessions on an ambulatory basis, analgesics with Diclofenac or Acetaminophen were prescribed for post-operative pain control.

Patients were evaluated at 4,12 and 24 weeks after the SWL session by KUB. Stone-free status was defined as the absence of radiographic residual stones in the follow-up imaging, any residual fragments visible on KUB was considered as non-stone-free. The KUB x-ray. Stone disintegration was defined as the presence of stone destruction, fragmentation or a decrease in size in the post-session KUB compared with the pre-session KUB. Retreatment was defined as the need for further surgical intervention (repeat SWL, rigid ureteroscopic lithotripsy (URSL), retrograde intrarenal surgery (RIRS) or percutaneous nephrolithotomy (PCNL)) for symptomatic patients or patients with residual stone fragments $>0.5 \mathrm{~cm}$. Complications were classified according to the Clavien-Dindo classification system. All data were analyzed and compared using t-tests and chi-squared tests with IBM SPSS statistics version 25.0 software. Statistical significance was set at $\mathrm{p}<0.05$.

The present study, including its research protocols and data collection, were approved by the Mackay Memorial Hospital Institutional Review Board. All personal information was de-identified prior to data analysis, thus ensuring patient data confidentiality.

\section{Results}

Of the 425 included patients, 209 were treated with USa SWL and 216 were treated with FS SWL. The mean age of patients was $53.3 \pm 13.2$ years in the USa group and $53.8 \pm 12.4$ years in the FS group $(\mathrm{p}=0.538)$. Comparison of the two groups revealed that the FS group was significantly more male dominated $(\mathrm{p}=0.016)$ and there were significantly larger stones in the USa group $(1.03 \pm 0.37 \mathrm{~cm}$ vs. $0.87 \pm 0.33 \mathrm{~cm}, \mathrm{p}<0.005)$ (Table 1$)$.

\section{Table 1 Patient demographics and stone characteristics}

\begin{tabular}{llll}
\hline Characteristic & USa & FS & p value \\
\hline Number of patients & 209 & 216 & \\
Patient age (mean \pm SD) & $53.3 \pm 13.2$ & $53.8 \pm 12.4$ & 0.568 \\
Patient gender & & & 0.016 \\
Male, n (\%) & $130(62.2 \%)$ & $158(73.1 \%)$ & \\
Female, n (\%) & $79(37.8 \%)$ & $58(26.8 \%)$ & \\
Stone size (cm) & $1.03 \pm 0.37$ & $0.87 \pm 0.33$ & 0.008 \\
$>1 \mathrm{~cm}(\mathrm{n})$ & 111 & 73 & \\
$<1 \mathrm{~cm}(\mathrm{n})$ & 98 & 143 & \\
\hline
\end{tabular}

Statistical analysis revealed better treatment outcomes in the USa group. The overall stone free rate was significantly better in the USa group compared with the FS group (USa $43.5 \%$ vs. FS $28.2 \%, \mathrm{p}<0.001$ ), and the overall stone disintegration rates were significantly higher (USa $85.6 \%$ vs. FS $64.3 \%, \mathrm{p}<0.001)$. Significant lower retreatment (USa $14.8 \%$ vs. FS $35.6 \%, \mathrm{p}<0.001$ ) and complication rates (USa $1.9 \%$ vs. FS $5.5 \%, \mathrm{p}=0.031$ ) were noted in the USa group compared with the FS group (Table 2).

Patients were further stratified by stone size $(<1 \mathrm{~cm}$ and $\geq 1 \mathrm{~cm}$ ), and the results were similar following stratification. In the group with renal stones $<1 \mathrm{~cm}$ the USa group had a significantly higher stone free rate (USa $56.1 \%$ vs. FS $33.5 \%, \mathrm{p}<0.001)$ and stone disintegration rate (USa $81.6 \%$ vs. FS $69.2 \%, \mathrm{p}=0.031$ ) compared with the FS group. The USa group also had a significantly lower retreatment rate (USa $7.1 \%$ vs. FS $26.5 \%, \mathrm{p}<0.001$ ) and complication rate (USa $1.0 \%$ vs. FS $6.9 \%, \mathrm{p}=0.029$ ) (Table 3).

In the group with stones $>1 \mathrm{~cm}$, the USa group still had a significantly better stone free rate (USa $32.4 \%$ vs. FS $17.8 \%, p=0.028$ ), stone disintegration rate (USa $89.2 \%$ vs. FS $54.8 \%, \mathrm{p}<0.001$ ) and retreatment rate (USa $21.6 \%$ vs. FS 53.4\%, p <0.001) compared with the FS group. However, the complication rates were not statistically different between the two groups (US $2.7 \%$ vs. FS $4.1 \%$, $\mathrm{p}=0.599)$, but the USa group did still have a lower complication ratio (Table 4).

The most common complication was post-SWL-related flank pain (Clavien-Dindo grade I), which occurred in $1(0.5 \%)$ USa group patient and $11(5.1 \%)$ FS group patients, however no cases required hospitalization. In all

Table 2 Overall outcomes, stone free rate, complication, retreatment rate

\begin{tabular}{lccr}
\hline & US $(\mathbf{n}=\mathbf{2 0 9})$ & FS $(\mathbf{n}=\mathbf{2 1 6})$ & p value \\
\hline Stone free rate (\%) & $91(43.5)$ & $61(28.2)$ & $<0.001$ \\
Disintegrate rate (\%) & $179(85.6)$ & $139(64.3)$ & $<0.001$ \\
Retreatment rate (\%) & $31(14.8)$ & $77(35.6)$ & $<0.001$ \\
Complication rate (\%) & $4(1.9)$ & $13(6.0)$ & 0.031 \\
\hline
\end{tabular}

Table 3 Stone size $<1 \mathrm{~cm}$, outcomes, stone free rate, complication, retreatment rate

\begin{tabular}{lclr}
\hline & US ( $\mathbf{n}=\mathbf{9 8})$ & FS ( $\mathbf{n = 1 4 3 )}$ & p value \\
\hline Stone free rate (\%) & $55(56.1)$ & $48(33.5)$ & $<0.001$ \\
Disintegrate rate (\%) & $80(81.6)$ & $99(69.2)$ & 0.031 \\
Retreatment rate (\%) & $7(7.1)$ & $38(26.5)$ & $<0.001$ \\
Complication rate (\%) & $1(1.0)$ & $10(6.9)$ & 0.029 \\
\hline
\end{tabular}


Table 4 Stone size $>1 \mathrm{~cm}$, outcomes, stone free rate, complication, retreatment rate

\begin{tabular}{lccr}
\hline & US $(\mathbf{n}=\mathbf{1 1 1})$ & FS $(\mathbf{n}=\mathbf{7 3})$ & p value \\
\hline Stone free rate (\%) & $36(32.4)$ & $13 /(17.8)$ & 0.028 \\
Disintegrate rate (\%) & $99(89.2)$ & $40(54.8)$ & $<0.001$ \\
Retreatment rate (\%) & $24(21.6)$ & $39(53.4)$ & $<0.001$ \\
Complication rate (\%) & $3(2.7)$ & $3(4.1)$ & 0.599 \\
\hline
\end{tabular}

cases symptoms were controlled with intravenous/intramuscular or oral non-steroidal anti-inflammatory drugs (NSAIDs). In the USa group, 1 patient developed ureteral stone street after SWL; the patient had a $1.7 \mathrm{~cm}$ renal stone and required further RIRS for obstruction release and residual stone removal (grade 3). Another patient had a urinary tract infection and required hospitalization for intravenous antibiotics (grade 2). In the FS group, 2 patients developed post-SWL ureteral stone street, and one required URSL for stone removal (grade 3); the other patient had stone passage during follow up (grade 2).

\section{Discussion}

SWL has been the primary treatment option for renal and upper urinary calculi since its introduction in the early 1980's [20]. Two major international urological associations: the European association of urology and the American urological association both recommend SWL as the treatment of choice for small to intermediate renal stones. However, the treatment has certain limitations which may predict poor treatment efficacy, including steep and narrow infundibulum, long lower pole calyx, and shockwave-resistant stones [21, 22].

To maximize the efficacy and outcomes while minimizing complications of SWL, certain options regarding patient and stone characteristics have been proposed [16]. Obese patients and those with a higher body mass index (BMI) may have a larger skin to stone distance revealed during clinical examination, which could lead to unfavorable SWL success rates [12, 23]. Some studies highlighted the importance of anatomical location and the architecture of the stones as measured by CT Hounsfield units [8-11].

Other evidence showed that during SWL sessions, the energy and frequency emitted by the lithotripter notably contributed to stone disintegration. It is recommended that the treatment strategy starts from a low energy level and low frequency rate, and then gradually increases. Starting from a low energy level has been shown to pre-sensitize the kidney and cause renal vasoconstrictions [13, 24], thus reducing renal damage and improving patient tolerance. Low frequency shock wave rates have been proven to be associated with better stone fragmentation in many previous studies [14, 25-27]. Although there is no gold standard for optimal treatment frequency, it has been shown that frequency rates of 60 shocks per minute $(1 \mathrm{~Hz})$ and 90 shocks per minute $(1.5 \mathrm{~Hz})$ both had better outcomes compared with 120 shocks per minute $(2 \mathrm{~Hz})$ [14, 25-27]. Decreasing both the initial energy and shock wave frequency results in a reduced total number of shock waves and energy being delivered, thus reducing the possible damage to the kidney, and therefore decreasing the possibility of complications and improving patient comfort. The patient could be more easily adapted to this treatment, thus minimizing patient movement and the use of sedative agents. In this way, energy delivery could be more focused on the target and stone disruption could be improved. The training and experience of the technicians and operators performing the procedure also has an impact on the success of an SWL session, studies have shown that the stone free rate improves as the operator completes a learning curve [28, 29].

In the current study, the SWL session starts with a low energy $(14 \mathrm{KV})$ and low frequency setting $(60 \mathrm{~Hz})$ in order to maximize the treatment effect. All of the SWL sessions were performed by the same two technicians who have over 10 years of experience. The authors consider this to be a strength of the current study. Using the same lowfrequency, low energy machine setup and having SWL sessions performed by the same two experienced operators, the SWL efficiency could be maximized while minimizing the inter-operator bias and learning curve bias.

Last but not least, the impact of the patient's stability during the SWL session must be considered. Patient movement and respiration during the SWL session can cause kidney movement [17], thus causing shock waves to be misdirected and focused away from the target. Excessive energy and inaccurate shockwaves may cause damage to the parenchyma and adjacent organs. Even when patients are under sedation or anesthesia during SWL, respiration related mistargeting remains inevitable. Constant tracking of the stone under a continuous fluoroscope could be the solution, however this may cause excessive ionizing radiation exposure. To overcome this problem, Chang et al. developed a real-time tracking ultrasound based system for renal stones [30]. In vitro and animal studies [31] showed the system to be efficient, and it could increase the accuracy of renal stone targeting and the efficiency of stone fragmentation.

The major advantage that ultrasound provides is realtime tracking of the stone without excessive radiation exposure to the patient. Although the dose of radiation during one SWL session may not be much and is not a worrisome dose compared to other invasive radiological procedures, the cumulative effect of ionizing radiation 
that needs to take into account includes the pre-treatment diagnostic imaging studies, such as CT or IVU, and possible repeat SWL treatment at a later date.

Chen et al. [19] reported on their clinical experience of renal stone treatment using an electromagnetic lithotripter integrated with an ultrasound-based real-time tracking system. Their results demonstrated increased accuracy of stone targeting with less shockwaves applied. Smith et al. [32] also compared the stone free rates between the USa localization technique and the traditional FS localization technique. The results revealed equivalent outcomes using the two different stone localization modalities but with the added benefit of no ionization exposure when using the USa technique. A randomized prospective study conducted by Van Besien et al. [33] demonstrated similar results, with the USa SWL stone-free rate not being inferior to the FS SWL, but with no need for ionizing radiation. This is probably most beneficial in pediatric patients, as children are 2-7 times more radiation-sensitive than adults [34]. In a study evaluating the outcomes and radiation exposure of children with cystine stones, Goren et al. [35] demonstrated that USa SWL was more effective and applied less ionizing radiation doses for pediatric patients. Also, in faintly radiopaque stones, such as cystine stones, USaguided SWL has better visualization of the stones than a fluoroscope.

In the current study, the USa group had a larger baseline stone size. Despite this, the USa group had a significantly better stone-free rate, stone-disintegration rate and a lower retreatment rate; this was regardless of stone size stratification. This result indicates better stone localization and targeting with the assistance of real-time ultrasound, which causes better stone fragmentation and secondarily leads to better stone clearance and lower retreatment rates. As for safety, the USa group had a significantly lower complication rate for smaller stones, and a lower complication rate for larger stones, although this did not reach statistical significance. These results further indicate the theoretical benefit of ultrasound tracking, that is, better accuracy leads to less surrounding tissue damage, which would be more significant in smaller targets.

Previously reported stone-free rate of ESWL ranged around 47 to $92 \%$ [2-7]. In our study, the main reason of our relative lower stone-free rates is because that stonefree status was more strictly defined. Stone-free status was defined as the absence of radiographic residual stones in the follow-up imaging, any residual fragments visible on KUB was considered as non-stone-free, even the fragments were less than $2 \mathrm{~mm}$ or $4 \mathrm{~mm}$, which was the most commonly used criteria in clinical practice and other studies.
Previous studies had certain limitations, such as small patient numbers or a lack of head to head comparisons [19, 32]. Despite its randomized prospective study design, Van Besein's study failed to show statistical significance between the different localization methods, this may be related to the small number of studied patients. A strength of the current study compared to others is that we have a large number of study patients, two similar energy source lithotripters using the same SWL protocol, and all procedures were performed by the same two experienced technicians, which provides strong head-to-head comparison while minimizing bias between different institutions and operators.

However, there were several limitations to the present study that need to be addressed. First, its retrospective study design limited the bias control, such as stone composition and anatomic parameters. Second, radiation exposure was not recorded besides self-reported preference by the two technicians. Third, CT was not routinely in every patient to assess factors that affect ESWL results, such as CT values, stone-to-skin distance and BMI. In addition, we compared two different electromagnetic lithotripters. Different lithotripters' acoustic output, efficiency, and collateral effects vary between models. Therefore, further strictly controlled studies are needed to support the conclusion. Lastly, SWL and localization techniques are very operatordependent. The current study was conducted in one single center and only two technicians operated the machine, thus minimizing inter-operator and institutional bias. Further studies could be conducted in a larger set of patients who are prospectively randomized.

\section{Conclusions}

SWL is a safe and non-invasive way of treating renal stones. this study compared two electromagnetic shock wave machines with different stone tracking systems. LiteMed LM-9200 ELMA lithotripter, which combined ultrasound and fluoroscopic stone targeting outperformed Medispec EM-1000 lithotripter, which used fluoroscopy for stone localization and tracking, with better stone-free rates and disintegration rates, as well as lower retreatment rates and complications with possible reduced radiation exposure.

\section{Abbreviations \\ CT: Computed tomography; SWL: Shock wave lithotripsy; KUB: Kidneys, ureter and bladder; IVU: Intravenous urography; URSL: Ureteroscopic lithotripsy; RIRS:} Retrograde intrarenal surgery; PCNL: Percutaneous nephrolithotomy.

\section{Acknowledgements}

Not applicable. 


\section{Authors' contributions}

THC: project development, data collection, data analysis, and manuscript writing. WRL: data collection and data analysis. WKT: data collection and data analysis. PKC: data collection and data analysis. MC: project development, data analysis, and manuscript editing. JST: project development, data analysis, and manuscript editing. AWC: manuscript editing. All authors have read and approved the manuscript.

\section{Funding}

None to declare.

\section{Availability of data and material}

Records and data pertaining to this study are in the patient's secure medical records in Mackay Memorial Hospital and are available from the corresponding author on reasonable request.

\section{Ethics approval and consent to participate}

The present study, including its research protocols and data collection, were approved by the Mackay Memorial Hospital Institutional Review Board. In view of the retrospective nature of the study, obtaining patients' informed consent was waived.

\section{Consent for publication}

Not applicable.

\section{Competing interests}

The authors declare that they have no competing interests.

\section{Author details}

${ }^{1}$ Department of Urology, MacKay Memorial Hospital, No. 92, Sec. 2, Zhongshan N. Rd., Taipei City 10449, Taiwan. ${ }^{2}$ Mackay Medical College, No.46, Sec. 3, Zhongzheng Rd., Sanzhi Dist., New Taipei City 252, Taiwan. ${ }^{3}$ Mackay Junior College of Medicine, Beitou District, Nursing, and Management, No.92, Shengjing Road, Taipei City 11272, Taiwan. ${ }^{4}$ School of Medicine, National Yang-Ming University, No.145, Zhengzhou Rd., Datong Dist., Taipei City 10341, Taiwan.

Received: 19 April 2020 Accepted: 31 October 2020 Published online: 10 November 2020

\section{References}

1. Chaussy C, Brendel W, Schmiedt E. Extracorporeally induced destruction of kidney stones by shock waves. Lancet (London, England). 1980;2(8207):1265-8.

2. Fialkov JM, Hedican SP, Fallon B. Reassessing the efficacy of the Dornier MFL-5000 lithotriptor. J Urol. 2000;164(3 Pt 1):640-3.

3. Nafie S, Dyer JE, Minhas JS, Mills JA, Khan MA. Efficacy of a mobile lithotripsy service: a one-year review of 222 patients. Scand J Urol. 2014;48(3):324-7.

4. Chung VY, Turney BW. The success of shock wave lithotripsy (SWL) in treating moderate-sized $(10-20 \mathrm{~mm})$ renal stones. Urolithiasis. 2016:44(5):441-4.

5. Nielsen TK, Jensen JB. Efficacy of commercialised extracorporeal shock wave lithotripsy service: a review of 589 renal stones. BMC Urol. 2017;17(1):59.

6. Abdel-Khalek M, Sheir KZ, Mokhtar AA, Eraky I, Kenawy M, Bazeed M. Prediction of success rate after extracorporeal shock-wave lithotripsy of renal stones-a multivariate analysis model. Scand J Urol Nephrol. 2004;38(2):161-7.

7. Lee CC, Lin WR, Hsu JM, Chow YC, Tsai WK, Chiang PK, Chen M, Chiu AW. Comparison of electrohydraulic and electromagnetic extracorporeal shock wave lithotriptors for upper urinary tract stones in a single center. World J Urol. 2019:37(5):931-5.

8. El-Nahas AR, El-Assmy AM, Mansour O, Sheir KZ. A prospective multivariate analysis of factors predicting stone disintegration by extracorporeal shock wave lithotripsy: the value of high-resolution noncontrast computed tomography. Eur Urol. 2007;51(6):1688-93 (discussion 1693-1684).
9. Nakasato T, Morita J, Ogawa Y. Evaluation of Hounsfield Units as a predictive factor for the outcome of extracorporeal shock wave lithotripsy and stone composition. Urolithiasis. 2015;43(1):69-75.

10. Cui HW, Devlies W, Ravenscroft S, Heers H, Freidin AJ, Cleveland RO, Ganeshan B, Turney BW. CT Texture analysis of ex vivo renal stones predicts ease of fragmentation with shockwave lithotripsy. J Endourol. 2017;31(7):694-700.

11. Williams JC Jr, Paterson RF, Kopecky KK, Lingeman JE, McAteer JA. High resolution detection of internal structure of renal calculi by helical computerized tomography. J Urol. 2002;167(1):322-6.

12. Patel T, Kozakowski K, Hruby G, Gupta M. Skin to stone distance is an independent predictor of stone-free status following shockwave lithotripsy. J Endourol. 2009;23(9):1383-5.

13. Handa RK, McAteer JA, Connors BA, Liu Z, Lingeman JE, Evan AP. Optimising an escalating shockwave amplitude treatment strategy to protect the kidney from injury during shockwave lithotripsy. BJU Int. 2012;110(11 Pt C):E1041-1047.

14. Pace KT, Ghiculete D, Harju M, Honey RJ. Shock wave lithotripsy at 60 or 120 shocks per minute: a randomized, double-blind trial. J Urol. 2005;174(2):595-9.

15. Davenport K, Minervini A, Keoghane S, Parkin J, Keeley FX, Timoney AG. Does rate matter? The results of a randomized controlled trial of 60 versus 120 shocks per minute for shock wave lithotripsy of renal calculi. J Urol. 2006;176(5):2055-8 (discussion 2058).

16. Kroczak T, Scotland KB, Chew B, Pace KT. Shockwave lithotripsy: techniques for improving outcomes. World J Urol. 2017;35(9):1341-6.

17. Suramo I, Paivansalo M, Myllyla V. Cranio-caudal movements of the liver, pancreas and kidneys in respiration. Acta Radiol. 1984;25(2):129-31.

18. Kuwahara M, Kambe K, Taguchi K, Saito T, Shirai S, Orikasa S. Initial experience using a new type extracorporeal lithotripter with an anti-misshot control device. J Lithotr Stone Dis. 1991;3(2):141-6.

19. Chen CJ, Hsu HC, Chung WS, Yu HJ. Clinical experience with ultrasoundbased real-time tracking lithotripsy in the single renal stone treatment. J Endourol. 2009;23(11):1811-5.

20. Chaussy C, Schmiedt E, Jocham D, Brendel W, Forssmann B, Walther V. First clinical experience with extracorporeally induced destruction of kidney stones by shock waves. J Urol. 1982;127(3):417-20.

21. Turk C, Petrik A, Sarica K, Seitz C, Skolarikos A, Straub M, Knoll T. EAU Guidelines on Interventional Treatment for Urolithiasis. Eur Urol. 2016;69(3):475-82.

22. Pradere B, Doizi S, Proietti S, Brachlow J, Traxer O. Evaluation of guidelines for surgical management of urolithiasis. J Urol. 2018;199(5):1267-71.

23. Wiesenthal JD, Ghiculete D, Ray AA, Honey RJ, Pace KT. A clinical nomogram to predict the successful shock wave lithotripsy of renal and ureteral calculi. J Urol. 2011;186(2):556-62.

24. Handa RK, Bailey MR, Paun M, Gao S, Connors BA, Willis LR, Evan AP. Pretreatment with low-energy shock waves induces renal vasoconstriction during standard shock wave lithotripsy (SWL): a treatment protocol known to reduce SWL-induced renal injury. BJU Int. 2009;103(9):1270-4.

25. Honey RJ, Schuler TD, Ghiculete D, Pace KT. A randomized, double-blind trial to compare shock wave frequencies of 60 and 120 shocks per minute for upper ureteral stones. J Urol. 2009;182(4):1418-23.

26. Li K, Lin T, Zhang C, Fan X, Xu K, Bi L, Han J, Huang H, Liu H, Dong W, et al. Optimal frequency of shock wave lithotripsy in urolithiasis treatment: a systematic review and meta-analysis of randomized controlled trials. J Urol. 2013;190(4):1260-7.

27. Yilmaz E, Batislam E, Basar M, Tuglu D, Mert C, Basar H. Optimal frequency in extracorporeal shock wave lithotripsy: prospective randomized study. Urology. 2005;66(6):1160-4.

28. Lee C, Best SL, Ugarte R, Monga M. Impact of learning curve on efficacy of shock wave lithotripsy. Radiol Technol. 2008;80(1):20-4.

29. Okada A, Yasui T, Taguchi K, Niimi K, Hirose Y, Hamamoto S, Ando R, Kubota Y, Umemoto Y, Tozawa K, et al. Impact of official technical training for urologists on the efficacy of shock wave lithotripsy. Urolithiasis. 2013;41(6):487-92.

30. Chang CC, Liang SM, Pu YR, Chen CH, Manousakas I, Chen TS, Kuo CL, Yu FM, Chu ZF. In vitro study of ultrasound based real-time tracking of renal stones for shock wave lithotripsy: part 1. J Urol. 2001;166(1):28-32.

31. Chang CC, Manousakas I, Pu YR, Liang SM, Chen CH, Chen TS, Yu FM, Yang WH, Tong YC, Kuo CL. In vitro study of ultrasound based real-time tracking 
for renal stones in shock wave lithotripsy: part II—a simulated animal experiment. J Urol. 2002;167(6):2594-7.

32. Smith HE, Bryant DA, KooNg J, Chapman RA, Lewis G. Extracorporeal shockwave lithotripsy without radiation: ultrasound localization is as effective as fluoroscopy. Urol Ann. 2016;8(4):454-7.

33. Van Besien J, Uvin P, Hermie I, Tailly T, Merckx L. Ultrasonography is not inferior to fluoroscopy to guide extracorporeal shock waves during treatment of renal and upper ureteric calculi: a randomized prospective study. Biomed Res Int. 2017:2017:7802672.

34. Richardson RB. Stem cell niches and other factors that influence the sensitivity of bone marrow to radiation-induced bone cancer and leukaemia in children and adults. Int J Radiat Biol. 2011;87(4):343-59.
35. Goren MR, Goren V, Ozer C. Ultrasound-guided shockwave lithotripsy reduces radiation exposure and has better outcomes for pediatric cystine stones. Urol Int. 2017;98(4):429-35.

\section{Publisher's Note}

Springer Nature remains neutral with regard to jurisdictional claims in published maps and institutional affiliations.
Ready to submit your research? Choose BMC and benefit from:

- fast, convenient online submission

- thorough peer review by experienced researchers in your field

- rapid publication on acceptance

- support for research data, including large and complex data types

- gold Open Access which fosters wider collaboration and increased citations

- maximum visibility for your research: over $100 \mathrm{M}$ website views per year

At BMC, research is always in progress.

Learn more biomedcentral.com/submissions 